\title{
Prävention und Ungewissheiten des Aufwachsens von Kindern
}

\author{
Biographisch frühe Versuche der Normalisierung von Lebensführung am Beispiel der Ernäh- \\ rungsprävention
}

Im Zuge wohlfahrtsstaatlicher Veränderungen hat die Prävention als Prinzip der Gestaltung von Kindheit an Bedeutung gewonnen. Die Prävention stellt ein Versuch dar, Ungewissheiten des Aufwachsens zu reduzieren. Wie das Beispiel der Ernährungsprävention zeigt, wird dieser Versuch zunehmend in der frühesten Lebensphase angesetzt und es kommt darüber zu einer Extension biographisch früher Normalisierungsversuche.

$\mathrm{D}$ as Aufwachsen von Kindern ist von einer Vielzahl an Ungewissheiten geprägt. Ungewiss ist, ob und wie sich pädagogische Bemühungen auf Kinder und deren Werden auswirken; ebenso ist ungewiss, welche Faktoren der Kindheit Einfluss auf das weitere Leben der Kinder haben. Entsprechend ist das Spektrum an Ungewissheiten, die z. B. Eltern hinsichtlich des Aufwachsens ihrer Kinder haben, breit. Es umfasst Fragen zur Einordnung von und Reaktion auf Äußerungen von Kindern, Fragen zur Strukturierung des kindlichen Alltags, aber auch Fragen zum Zeitpunkt und Art der außerfamilialen Betreuung, zur Förderung der Entwicklungs-, Lern- und Bildungsprozesse oder zur prospektiven Lebensführungsweise. Auf letzteren Aspekt wird

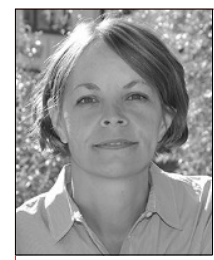

\section{Friederike Schmidt}

Universität Siegen, Siegen, Deutschland

*1976; PD Dr.in phil.; Erziehungswissenschaftlerin mit

Schwerpunkten in der Kindheits- und Jugendforschung, (Sozial-)

Pädagogischen Wissensbildung, Reflexiven Pädagogischen

Anthropologie und Rekonstruktiven Sozialforschung; seit 2020

Akademische Rätin an der Universität Siegen.

friederike.schmidt@uni-siegen.de

Zusammenfassung Präventive Versuche der Reduktion von Ungewissheiten haben in den letzten Jahrzehnten an Bedeutung gewonnen. Das Beispiel der Ernährungsprävention zeigt, dass zunehmend in der frühesten Lebensphase von Kindern angesetzt wird. Darüber gewinnen biographisch frühe Versuche der Normalisierung von Subjekten und deren Lebensführung an Relevanz.

Schlüsselwörter Prävention, Ungewissheit, Normalisierung, Kindheit, Ernährung im Folgenden am Beispiel der Ernährung von Kindern eingegangen.

\section{Prävention und Ungewissheiten des Aufwachsens} von Kindern

Die jeweilige Ausrichtung und das Erleben von Ungewissheiten sind individuell gelagert, lassen sich darauf aber nicht reduzieren. Vielmehr sind sie grundlegend in historisch-kulturelle und gesellschaftliche Zusammenhänge eingelassen. Für gegenwärtige Ungewissheiten hinsichtlich des Aufwachsens sind u. a. wohlfahrtsstaatliche Veränderungen von Bedeutung, die sich Ende der 1990er-Jahre etabliert haben. In deren Rahmen gewinnen soziale Investitionen an Bedeutung und werden präventive Bemühungen in der Kindheit relevant (vgl. Mierendorff 2018). Eine Zunahme kindbezogener Präventionsmaßnahmen zeigt sich u. a. im 13. Kinderund Jugendbericht mit dessen Schwerpunkt auf „Mehr Chancen für gesundes Aufwachsen - Gesundheitsbezogene Prävention und Gesundheitsförderung in der Kinder- und Jugendhilfe" (BMFSFJ 2009) und in frühpädagogischen Bildungsplänen, die in unterschiedlicher Akzentuierung auf Präventionsmaßnahmen setzen.

Der Beitrag knüpft an diese Beobachtungen an. Er geht von der Grundannahme aus, dass die Zunahme an Prävention, die in den letzten Jahrzehnten zu beobachten ist, Effekte auf den Umgang mit Ungewissheiten hinsichtlich des Aufwachsens von Kindern hat. Dabei lässt sich Prävention zunächst als wissensbasierter Versuch der Bearbeitung von Ungewissheiten ausweisen. Der Prävention liegen statistische Daten zum Auftreten sozialer Phänomene und deren Zusammenhänge zugrunde, auf deren Basis Prognosen über die Zukunft getrof- 
fen werden. Als Reaktionen auf eine erwartete Zukunft greift die Prävention jedoch einer Entwicklung voraus, von der man zum Zeitpunkt der Einflussnahme noch nicht weiß, ob, und wenn ja, wie sie sich ausbildet. Auch bleibt offen, ob die präventiven Bemühungen erfolgreich sind und welchen Anteil diese an der jeweils eingetroffenen Zukunft haben (Bröckling 2004). Denn, ob eine Präventionsmaßnahme z. B. verhindert, dass sich das Kind später ungesund ernährt oder hohes Körpergewicht entwickelt, lässt sich kaum en détail klären, ist das zukünftig Geschehene stets von weiteren und immer auch unvorhersehbaren Faktoren beeinflusst.

Die Prävention ist somit grundlegend von Ungewissheiten bestimmt. Dessen ungeachtet besitzen Präventionsmaßnahmen eine hohe Plausibilität als Versuche der Reduktion von Ungewissheiten: Sie schließen mit ihrem Bemühen einer vorsorglichen Verhinderung von Problemen an ein Alltagswissen an, dass sich Probleme frühzeitig besser bearbeiten lassen (ebd.). Gerade Wissensbestände zu statistischen Verteilungen von Phänomenen entfalten eine Überzeugungskraft, da sie erklärungsbedürftig machen, warum trotz des Wissens über Zusammenhänge nicht darauf Einfluss genommen wird. Im Falle der kindbezogenen Ernährungsprävention kommt hinzu, dass mit der Zielgruppe „Kinder“ an ein modernes Zukunftsversprechen sowie mit dem Ziel der Verbesserung von Gesundheit und Leistung an Leitnormen (spät-)moderner Gesellschaften angeschlossen wird.

Im Lichte dieser Plausibilitäten wird Prävention als Prinzip der Gestaltung des Aufwachsens von Kindern zunehmend relevant. Das Beispiel der Ernährungsprävention zeigt, dass hierbei vermehrt die früheste Lebensphase in den Fokus rückt und darüber zu einer biografisch frühen Normalisierung der Subjekte und deren Lebensführung angesetzt wird. In der Darlegung dieses Befunds wird nachfolgend zunächst auf eine Intensivierung der gesellschaftlichen Aufmerksamkeiten für die Ernährung von Kindern eingegangen. Teil dessen ist eine Zunahme der Ernährungsprävention in der frühesten Lebensphase von Kindern, wodurch Fragen der Konfiguration von Ungewissheiten in und durch Präventionsmaßnahmen in den Blick geraten. Der Beitrag schließt mit einem Fazit, in dem die analytischen Befunde kritisch reflektiert werden.

\section{Ernährungsrisiken von Kindern im Fokus gesell- schaftlicher Aufmerksamkeit}

Mit dem Aufkommen des Sozialinvestitionsstaats (Lessenich 2008) hat das öffentliche und politische Interesse an den Bedingungen und Risiken des Aufwachsens von Kindern zugenommen (Betz und Bischoff 2013). Verdichtet zeigt sich das bei der Ernährung von Kindern:
Dies wird zum Ersten an einem Anstieg an evaluativen Untersuchungen zur institutionellen Verpflegung von Kindern deutlich. Weitgehend dem Bereich der Ernährungswissenschaft zugeordnet, weisen die Studien darauf hin, dass die Mehrzahl der pädagogischen Institutionen kein gesundes Essensangebot für Kinder und Jugendliche bereitstellt (ex. Tecklenburg et al. 2016). Entsprechend werden Modellvorschläge für die Nahrungsversorgung in pädagogischen Einrichtungen entwickelt. Parallel dazu werden vermehrt elementarpädagogische Konzepte mit gesundheitsbezogenem Schwerpunkt im Auftrag von Krankenkassen oder durch Bildungsstiftungen veröffentlicht (Bertelsmann Stiftung 2012; Voss und Viernickel 2016).

Zum Zweiten ist seit knapp 20 Jahren ein Anstieg der Forschung zum Ernährungs- und Gesundheitszustand von Kindern in Deutschland festzustellen. Breit rezipiert sind die in den Studien diagnostizierten Ernährungsund Übergewichtsprobleme junger Menschen (Mensink et al. 2007). Wenn auch, den Studien folgend, nur ein Teil der Kinder von Ernährungs-, Übergewichts- und Gesundheitsproblemen betroffen ist, wird öffentlich und politisch die Ernährung von Kindern zunehmend und vornehmlich mit Bezug auf Ernährungs- und Übergewichtsprobleme erfasst. Zentraler Referenzpunkt der Debatten bilden Annahmen einer „Adipositas-Epidemie“ (Branca et al. 2007, S. XI) in Europa, zu denen u. a. die Weltgesundheitsorganisation (WHO) gelangt (kritisch dazu u. a. Schorb 2015).

\section{Konfiguration von Ungewissheiten in und durch die Ernährungsprävention}

Insgesamt ist seit Anfang der 2000er-Jahre eine Verdichtung gesundheitspolitischer Bemühungen in Deutschland festzustellen. Eine zentrale Relevanz kommt dem sog. Nationalen Aktionsplan „IN FORM - Deutschlands Initiative für gesunde Ernährung und mehr Bewegung “ zu, mit dem seit 2008 gesundheitspolitische Bemühungen zur Ernährung und Bewegung von Kindern gebündelt werden. Zentrales Motiv der Initiative ist das Vorhaben, dass Menschen „gesünder aufwachsen und von einer höheren Lebensqualität und einer gesteigerten Leistungsfähigkeit in Bildung, Beruf und Privatleben profitieren“ (IN FORM 2021, o. S.). Neben gesundheitsbezogenen Themen geht es in der Initiative somit auch um leistungsbezogene Probleme, denen präventiv zu begegnen ist (IN FORM 2017, S. 5). In diesen Versuchen wird v. a. die jüngste Lebensphase von Kindern fokussiert. Man kann insofern von Bemühungen einer biographisch frühen Bewältigung von Ungewissheiten sowie einer vorsorglichen Normalisierung der Lebens- 
führung von Kindern sowie ihren Erziehungs- und Sorgeverantwortlichen sprechen.

\section{Bemühungen einer biographisch frühen Bewältigung von Ungewissheiten}

Die IN FORM-Initiative zielt auf alle Lebensphasen ab, v. a. fokussiert wird aber die Kindheit (Schmidt 2020). Denn - so etwa die Beschreibung der App „Kind \& Essen“, die für Eltern zur Unterstützung der Ernährung ihrer Kinder konzipiert wurde: „Das Kleinkindalter ist entscheidend für die Basis eines gesunden Ess- und Ernährungsverhaltens, für prägende Geschmackserlebnisse und erste, wichtige Entwicklungsschritte" (IN FORM 2019). Hier greift ein Verständnis menschlicher Entwicklung, dem folgend das Werden eines Menschen durch die Kindheit vorstrukturiert wird, da in dieser Lebensphase die entscheidenden Grundlagen gelegt werden. Wird in dieser Lebensphase nicht entsprechend reagiert und den Kindern die „richtigen“ Stoffe zugeführt, fehlt - so die Logik - der Grundstock für eine gesunde Entwicklung und Leistungsfähigkeit der Kinder. Damit bleibt ungewiss, wie sich die Subjekte im Weiteren entwickeln und welches Gesundheits- und Leistungsverhalten sie später zeigen.

Bemühungen einer biographisch frühen Reduktion von Ungewissheiten hinsichtlich der Gesundheit und des Leistungspotentials der Subjekte zeigen sich auch darin, dass bereits auf werdende Mütter präventiv eingewirkt wird. Die Schwangerschaft wird in der Initiative neben der Geburt als „eine sensible Phase“ (IN FORM 2017, S. 18) ausgewiesen, „um die Gesundheit des Kindes nicht nur kurz-, sondern auch langfristig zu fördern. Bereits in der Schwangerschaft werden Weichen für spätere gesundheitliche Entwicklungen gestellt“" (ebd.). Entsprechend gilt es, diese Phase über präventive Maßnahmen zu bearbeiten (Schmidt 2021). Diese Bemühungen, Ungewissheiten hinsichtlich der Gesundheitsentwicklung von Subjekten dadurch zu reduzieren, dass pränatal eingegriffen und spezifischen Entwicklungen vorgegriffen wird, führt letztlich zum Paradox, dass sich Ungewissheiten vergrößern. Denn mit jedem früheren Eingriff in die Entwicklung der Subjekte erweitert sich die Spanne zwischen Eingriff und angestrebtem - wenngleich niemals erreichbarem (Bröckling 2012, S. 95) - Zustand.

Diese Zunahme an Ungewissheiten wird in den Projekten der IN FORM-Initiative kaum thematisiert. Vielmehr werden Ungewissheiten darüber verschleiert, dass wiederholt auf die Notwendigkeit eines frühen Eingriffs verwiesen wird. Zudem werden, an Extremfolgen veranschaulicht, Zusammenhänge zwischen aktuellem Verhalten und zukünftigen negativen Entwicklungen her- gestellt - so etwa, wenn in der Dokumentation zu der Präventionsmaßnahme „9+12 - Gemeinsam gesund in Schwangerschaft und erstem Lebensjahr " (im Weiteren: „9 + 12“) der Plattform Ernährung und Bewegung e.V. (peb) deutlich gemacht wird, dass „das Ernährungsund Bewegungsverhalten während der Schwangerschaft und im Säuglingsalter [...] einen dauerhaften und prägenden Einfluss auf die spätere (Über-)Gewichtsentwicklung sowie auf das Risiko assoziierter Erkrankungen wie Diabetes mellitus Typ 2 und kardiovaskuläre Erkrankungen“ (peb 2014, S. 18) haben. Man kann hier auch von einem „aktivistischen Negativismus“ (Bröckling 2012, S. 93 f.) sprechen, welcher der Prävention grundlegend ist. Dem folgend bildet die „Vermeidung künftiger Übel [...] die Stoßrichtung vorbeugender Anstrengungen “ (ebd., S. 94), über die sich wiederum eine Vielzahl fortwährender Bemühungen der Vorbeugung aktivieren lassen. Schließlich „kann man nie genug und nie früh genug [vorbeugen]“ (ebd., S. 95).

\section{Vorsorgliche Normalisierungen der Lebensführung}

Präventionsmaßnahmen basieren auf statistischer Datenerhebung und -aggregierung. Die sich zeigenden Verteilungen empirischer Phänomene und deren Differenzierung in sog. Felder des Normalen und Abweichenden bilden die Grundlage für Prognosen über zukünftige gesellschaftliche Entwicklungen, angesichts derer präventiv gehandelt wird. In diesem Sinne folgt die (Ernährungs-)Prävention grundlegend einer normalisierenden Logik. Die darüber erfolgten Interventionen lassen sich so auch als Versuche einer Normalisierung von Lebensführung näher beschreiben.

An normalismustheoretische Arbeiten anschließend kann die Prävention in Relation zu der von Link bezeichneten „normalistische[n] Urangst, nicht normal zu sein“ (Link 2009, S. 246), gestellt werden. Die Frage, ob etwas normal ist oder nicht, lässt sich als spezifische und grundlegende Ungewissheit (spät-)moderner Gesellschaften begreifen, in denen Menschen als sich selbst-beobachtend und sich selbst-regulierend aufgerufen sind und dabei „ihre Ernährung, ihr Sexualleben, ihren Erziehungsstil usw. in die Grenzen der „Normalität" einbinden, sich notfalls therapieren lassen, um das zu erreichen “ (Hahn 2003, S. 30). Zwar lassen sich gegenwärtige normalistische Ungewissheiten nicht alleine auf eine Denormalisierungsangst engführen. Gegenwärtig zu beobachten ist auch eine „normalistische Lust, die beständig oszilliert zwischen dem Drang zur Erkundung des Grenzraums der Normalität (...) und der Rückkehr in die durch den „Bauch“ der Gaußkurve symbolisierbare Mitte“ (Balke 2003, S. 145). Im Falle der Prävention ist jedoch festzustellen, dass sich diese wesentlich 
über eine Problematisierung des Ausreizens des Normalfeldes begründet. Negativer Horizont, auf den die Subjekte in den Ernährungspräventionsmaßnahmen wiederkehrend hingewiesen werden, sind Ernährungsund Übergewichtsprobleme, die als Abweichungen vom Normalfeld markiert werden und die es zu vermeiden gilt. Insofern ist es naheliegend, dass die (Ernährungs-) Prävention an Denormalisierungsängste anschließt bzw. diese, sofern sie nicht bestehen, aktiviert.

Dabei ist festzustellen, dass in Maßnahmen der Ernährungsprävention auch Bezüge zur Differenz normal/abweichend dezidiert hergestellt werden. Beispielhaft dafür ist das zuvor genannte Präventionsprojekt „,9+12“, in dem auf Mütter während der Schwangerschaft und dem ersten Lebensjahr ihres Kindes eingewirkt wird mit dem Ziel einer Verbesserung der Gesundheit von Kindern. Ein zentrales Element der Präventionsmaßnahme bildet der sog. Präventionspass, der als Äquivalent zu den Vorsorgeuntersuchungsheften der sog. U-Untersuchungen entwickelt worden ist. In dem Pass wird „das Gesundheitsverhalten der (werdenden) Mütter“ (peb o. A., S. 9) tabellarisch dokumentiert und dieses entlang der Differenz normal/abweichend bzw. normal/,, behandlungsbedürftig“ erfasst - so etwa, wenn festgehalten wird, ob der BMI-Wert oder die Gewichtszunahme der Mütter während der Schwangerschaft „normal“, „grenzwertig“ oder „behandlungsbedürftig“ (ebd.) ist.

\section{Fazit}

Präventive Bemühungen sind mit dem Paradox konfrontiert, eine Zukunft vorwegzunehmen, von der nicht gewusst werden kann, ob sie eintritt. Zugleich wird eine Zukunft angestrebt, bei der zum Zeitpunkt der präventiven Intervention ungewiss bleibt, ob sie erreicht wird und welchen Anteil der Prävention an der jeweils eingetroffenen Zukunft dabei zukommt. Diese Ungewissheiten verschärfen sich dann, wenn präventive Maßnahmen biographisch früh einsetzen. Denn damit erweitern sich Möglichkeiten weiterer Einflussfaktoren auf das jeweils Angestrebte. Ungewissheiten spielen in Präventionsmaßnahmen bzw. in den Programmen zu diesen dennoch keine zentrale Rolle. Sie werden kaum thematisiert. Stattdessen werden sie über wiederkehrende Verweise auf die (erwarteten) Wirkungen der Prävention sowie Hinweisen auf Zusammenhänge zwischen gegenwärtigem Verhalten und zukünftiger Entwicklung verschleiert.

Der wiederkehrende Verweis auf das, was passieren kann und was präventiv erreicht werden könnte, gibt sowohl Ungewissheiten als auch kritischen Rückfragen an Präventionsmaßnahmen wenig Raum. Vielmehr werden darüber die Möglichkeiten sowie Notwendigkei- ten der Prävention plausibilisiert. Dabei ist zu beachten, dass sich Vorsorgelogiken in den letzten Jahrzehnten als eine „dominante Ratio [etabliert haben; F. S.], unter der zeitgenössische [kapitalistisch organisierte; F. S.] Gesellschaften ihr Verhältnis zur Zukunft verhandeln und organisieren " (Bröckling 2012, S. 93). Jedoch ist eine kritische Reflexion präventiver Bemühungen mindestens aus drei Gründen angebracht:

- Zum Ersten greifen Präventionsmaßnahmen vorsorglich in das Geschehen ein, intervenieren also bevor Probleme da sind und fordern zu spezifischen Lebensführungsweisen auf (Dollinger 2006; Ziegler 2001). In der Konsequenz führt die Prävention zu einer normalisierenden Regulierung der Lebensführung, die zunehmend biographisch früh auf Dauer gestellt wird. Dies wird - im Falle der Ernährungsprävention - nicht nur der individuellen und sozio-kulturellen Heterogenität der Ernährungsweisen kaum gerecht, sondern trägt auch der körperlichen Diversität nicht hinreichend Rechnung (Rose und Schorb 2017). Zudem bleibt vernachlässigt, dass aus „ungesundem“ Essen und hohem Körpergewicht nicht zwangsläufig Gesundheits- und Leistungsprobleme resultieren müssen bzw. dass „gesundes“ Essen und ein schlanker Körper nicht zwingend zu mehr Gesundheit und Leistung führen - zumal auch Gesundheit und Leistung historisch-kulturelle und gesellschaftliche Phänomene darstellen (Hafen 2014).

- Zum Zweiten werden Ernährung und in der Folge die Lebensphase der Kindheit mit der Verdichtung von Präventionsmaßnahmen einer Verwertungslogik unterstellt, die in sozialinvestiver Manier biographisch früh aktiviert wird. Hiernach wird ernährt, um gesund zu sein und mehr zu leisten. Dass Essen und Ernährung multipel bedeutsam sind, indem z. B. Essen ein Medium lebensgeschichtlicher Erfahrungen darstellt oder mit dem Essen Erfahrungen von Genuss und Ästhetik gekoppelt werden, bleibt in der Ernährungsprävention weitgehend unbeachtet. Stattdessen werden diese Aspekte nur dann bedeutsam, wenn sie sich zur Herstellung von (mehr) Gesundheit und Leistung nutzen lassen.

- Eng damit verbunden leistet die Ernährungsprävention zum Dritten eine Rationalisierung und Moralisierung von Ernährung und dabei von Lebensführung Vorschub (Barlösius 2014). Mit dem umfänglichen und biographisch frühen Einwirken ist der Gedanke verbunden, dass die Subjekte nur hinreichend aufgeklärt werden müssen, damit sie sich ernährungswissenschaftlichen Maßgaben folgend "gut" und „richtig“ ernähren. Dass aber Essen als soziales Totalphänomen (Mauss 1975) nicht nur eine Frage von 
Intention und Wissen ist, bleibt vernachlässigt. Ebenfalls kaum berücksichtigt sind die sozial ungleichen Ernährungsmöglichkeiten. Stattdessen werden die frühzeitig aufgeklärten Subjekte für möglicherweise auftretende individuelle und gesellschaftliche Ernährungs- und Gesundheitsprobleme verantwortlich gemacht.

Vor dem Hintergrund dieser kritischen Einwände auf präventive Bemühungen zu verzichten, scheint dennoch wenig überzeugend. Dass Präventionsmaßnahmen Probleme verhindern und die Gesundheit der Subjekte verbessern können, ist unbestritten - Krebsvorsorgeuntersuchungen oder die gegenwärtige pandemische Lage zeigen dies eindrücklich. Jedoch wäre in Bezug auf die (Ernährungs-)Prävention eine kritische Distanznahme $\mathrm{zu}$ wünschen, entfaltet diese - mindestens im Kontext der Ernährung von Kindern - eine geradezu selbstgeläufige Plausibilität, welche die angesprochenen problematischen Nebeneffekte präventiver Bemühungen vielfach verdeckt.

Eingegangen. 28. September 2021

Angenommen. 15. November 2021

Funding. Open Access funding enabled and organized by Projekt DEAL.

Open Access. Dieser Artikel wird unter der Creative Commons Namensnennung 4.0 International Lizenz veröffentlicht, welche die Nutzung, Vervielfältigung, Bearbeitung, Verbreitung und Wiedergabe in jeglichem Medium und Format erlaubt, sofern Sie den/die ursprünglichen Autor(en) und die Quelle ordnungsgemäß nennen, einen Link zur Creative Commons Lizenz beifügen und angeben, ob Änderungen vorgenommen wurden.

Die in diesem Artikel enthaltenen Bilder und sonstiges Drittmaterial unterliegen ebenfalls der genannten Creative Commons Lizenz, sofern sich aus der Abbildungslegende nichts anderes ergibt. Sofern das betreffende Material nicht unter der genannten Creative Commons Lizenz steht und die betreffende Handlung nicht nach gesetzlichen Vorschriften erlaubt ist, ist für die oben aufgeführten Weiterverwendungen des Materials die Einwilligung des jeweiligen Rechteinhabers einzuholen.

Weitere Details zur Lizenz entnehmen Sie bitte der Lizenzinformation auf http://creativecommons.org/licenses/by/4.0/deed.de.

\section{Literatur}

Balke, F. (2003). Der Zwang des „Habitus“. In J. Link, T. Loer \& H. Neuendorff (Hrsg.), „Normalität“" im Diskursnetz soziologischer Begriffe (S. 135-149). Heidelberg: Synchron.

Barlösius, E. (2014). Dicksein. Frankfurt am Main, New York: Campus.

Bertelsmann-Stiftung (2012). Die gute gesunde Kita gestalten. https:// www.bertelsmann-stiftung.de/de/publikationen/publikation/did/die-gute-gesunde-kita-gestalten-referenzrahmen/. Zugegriffen: 18. Okt. 2021.
Betz, T., \& Bischoff, S. (2013). Risikokind und Risiko Kind. In H. Kelle \& J. Mierendorff (Hrsg.), Normierung und Normalisierung der Kindheit (S. 60-81). Weinheim, Basel: Beltz Juventa.

BMFSFJ (2009). 13. Kinder- und Jugendbericht. https://www.bmfsfj.de/ bmfsfj/service/publikationen/13--kinder--und-jugendbericht/87246. Zugegriffen: 26. Sept. 2021.

Branca, F., Nikogosian, H., \& Lobstein, T. (Hrsg.). (2007). Die Herausforderung Adipositas und Strategien zu ibrer Bekämpfung in der Europäischen Region der WHO. Kopenhagen: WHO Regionalbüro für Europa.

Bröckling, U. (2004). Prävention. In U. Bröckling, S. Krasmann \& T. Lemke (Hrsg.), Glossar der Gegenwart (S. 210-215). Frankfurt am Main: Suhrkamp.

Bröckling, U. (2012). Dispositive der Vorbeugung. In C. Daase, P. Offermann \& V. Rauer (Hrsg.), Sicherheitskulturen. Soziale und politische Praktiken der Gefahrenabwehr (S. 93-108). Frankfurt am Main, New York: Campus.

Dollinger, B. (2006). Prävention. In B. Dollinger \& J. Raithel (Hrsg.), $A k$ tivierende Sozialpädagogik. Ein kritisches Glossar (S. 145-154). Wiesbaden: VS.

Hafen, M. (2014). Mythologie der Gesundheit. Heidelberg: Carl Auer.

Hahn, A. (2003). Aufmerksamkeit und Normalität. In J. Link, T. Loer \& H. Neuendorff (Hrsg.), „Normalität“ im Diskursnetz soziologischer Begriffe (S. 23-37). Heidelberg: Synchron.

IN FORM (2017). Deutschlands Initiative für gesunde Ernährung und mehr Bewegung. https://www.in-form.de/in-form/in-form-zwischenbericht/. Zugegriffen: 17. Sept. 2021.

IN FORM (2019). App „Kind \& Essen“. https://www.in-form.de/netzwerk/projekte/app-kind-essen/. Zugegriffen: 17. Sept. 2021.

IN FORM (2021). Die Initiative IN FORM. https://www.in-form.de/inform/allgemein/. Zugegriffen: 10. Nov. 2021.

Lessenich, S. (2008). Die Neuerfindung des Sozialen. Bielefeld: transcript.

Link, J. (2009). Versuch über den Normalismus. Göttingen: Vandenhoeck \& Ruprecht.

Mauss, M. (1975). Soziologie und Anthropologie. Bd. 2. München, Wien: Carl Hanser.

Mensink, G., et al. (2007). Forschungsbericht. https://www.rki.de/DE/ Content/Gesundheitsmonitoring/Studien/Kiggs/Basiserhebung/Eskimo/eskimo_node.html. Zugegriffen: 1. Okt. 2021.

Mierendorff, J. (2018). Potenziale eines wohlfahrtsstaatstheoretischen $\mathrm{Zu}$ ganges in der Kindheitsforschung. In T. Betz, et al. (Hrsg.), Institutionalisierungen von Kindheit (S. 129-145). Weinheim, Basel: Beltz Juventa.

Plattform Ernährung und Bewegung e. V. (peb) (2014). Dokumentation zum Projekt ",9+12. Gemeinsam gesund in Schwangerschaft und erstem Lebensjahr". "https://www.pebonline.de/fileadmin/pebonline/Projekte/9_12/ Reader_9_12.pdf. Zugegriffen: 22. Okt. 2021.

Plattform Ernährung und Bewegung e. V. (peb) (o. A.). Präventionspass. Ernährung und Bewegung. https://www.pebonline.de/fileadmin/pebonline/ Projekte/9_12/peb_9_12_Broschuere_Praeventionspass_07.pdf. Zugegriffen: 22. Okt. 2021

Rose, L., \& Schorb, F. (Hrsg.). (2017). Fat Studies in Deutschland. Weinheim, München: Beltz Juventa.

Schmidt, F. (2020). In Sorge ums Kind. In C. Dietrich, O. Sanders, N. Uhlendorf \& F. Beiler (Hrsg.), Anthropologie der Sorge (S. 202-212). Weinheim, München: Beltz Juventa.

Schmidt, F. (2021). Konfigurationen von Ängsten und Unsicherheiten in präventiven Gesundheitsmaßnahmen. In C. Thompson, J. Zirfas, W. Meseth \& T. Fuchs (Hrsg.), Erziehungswirklichkeiten in Zeiten von Angst und Verunsicherung (S. 102-116). Weinheim, Basel: Beltz Juventa.

Schorb, F. (2015). Die Adipositas-Epidemie als politisches Problem. Wiesbaden: Springer VS.

Tecklenburg, E., Arens-Azevêdo, U., \& Pfannes, U. (2016). Verpflegung in Kindertageseinrichtungen (VeKiTa). Ernährungsumschau, 63, 48-55.

Voss, A., \& Viernickel, S. (2016). Gute gesunde Kita. Berlin: das netz.

Ziegler, H. (2001). Prävention. Widersprüche, 21, 7-24. 
Hier steht eine Anzeige.

\section{Springer}

